\title{
NOTAS CARIOLÓGICAS SOBRE ALGUNAS ESPECIES NORTEAFRICANAS
}

\author{
Emilio RUIZ DE CLAVIJO
}

\begin{abstract}
RESUMEN. En esta nota dan a conocer 13 recuentos cromosómicos realizados en plantas procedentes de Marruecos, confirmándose los números cromosómicos de cinco endemismos norteafricanos o ibero-norteafricanos poco conocidos desde el punto de vista cariológico: Cistus heterophyllus Desf., $\mathrm{n}=9$; Tuberaria macrosepala (Cosson) Willk., $\mathrm{n}=18$; Psychine stylosa Desf. var. typica Maire, $\mathrm{n}=15$; Stachys circinata L'Her., $\mathrm{n}=10$ y Antirrhinum ramosissimum Cosson \& Durieu, $\mathrm{n}=8$.
\end{abstract}

Palabras clave. Cariología, Marruecos.

SUMMARY. The chromosome number of 13 species from Morocco are reported in this paper. The number of five of them, north-african, or ibero north-african little know from the cariological point of view endemisms, (Cistus heterophyllus Desf., $\mathrm{n}=9$; Tuberaria macrosepala (Cosson) Willk., $\mathrm{n}=18$; Psychine stylosa Desf. var. typica Maire, $\mathrm{n}=15$; Stachys circinata L'Her., $\mathrm{n}=10$ y Antirrhinum ramosissimum Cosson \& Durieu, $\mathrm{n}=8$ ) are confirmed.

Key words. Cariology, Morocco

Para el estudio cariológico se han utilizado botones florales procedentes de plantas silvestres fijados con etanol y ácido acético (3:1), realizándose la tinción según la técnica de Snow (1963). Los pliegos testigo se encuentran depositados en el herbario del Departamento de Biología Vegetal y Ecología de la Facultad de Ciencias, Universidad de Córdoba.

Cistus heterophyllus Desf., Fl. Atl. 1:411 (1798)

$n=9$ (fig.1)

Material estudiado. MARRUECOS: Entre Alhoceima y Targuist, 12-V-1989, B. Cabezudo, E. Domínguez, E. Ruiz de Clavijo et al.(COFC 19868).

Confirmamos el único recuento previo que conocemos de este taxon, dado por Dansereau (1940,sec. Fedorov,1969:222), para material cuya procedencia no indica.

Cistus salvifolius L., Sp. Pl. 524 (1753)

$n=9$ (fig.2)

Material estudiado. MARRUECOS: Entre Alhoceima y Targuist, 12-V-1989, B. 

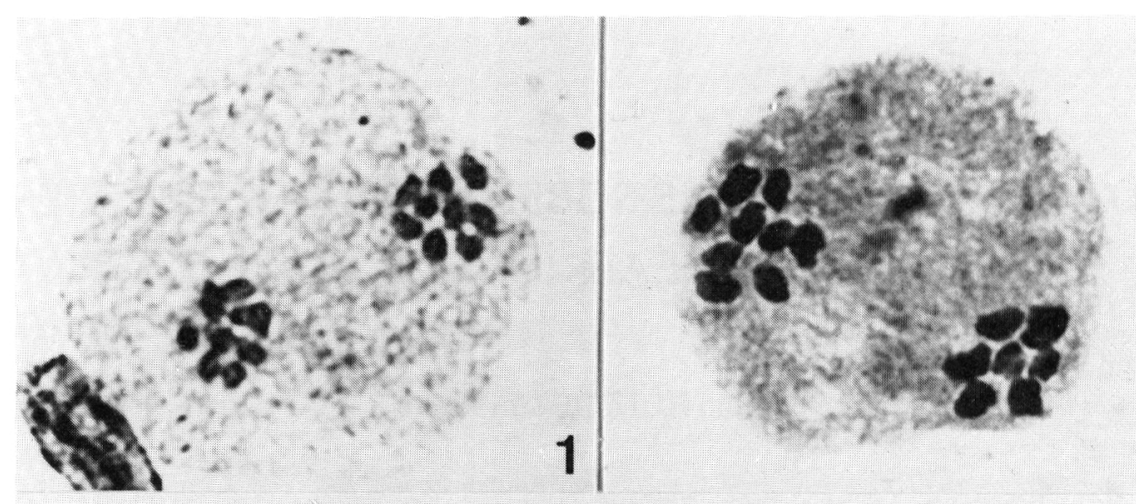

2
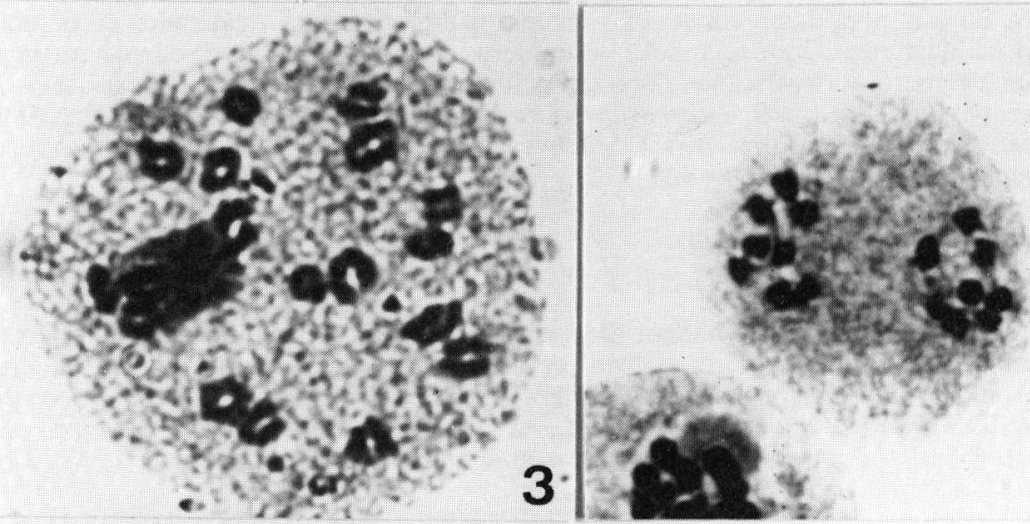

4
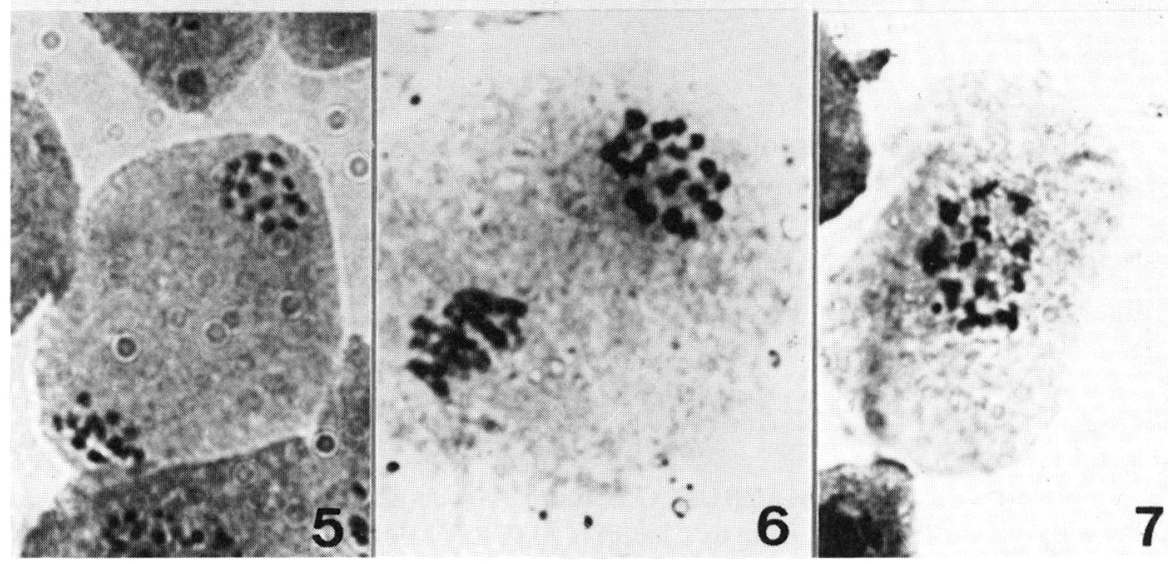

Fig. 1- Anafase I de Cistus heterophyllus Desf., n=9. Fig. 2- Anafase I de Cistus salvifolius L., $n=9$. Fig. 3- Diacinesis de Tuberaria macrosepala (Cosson) Willk., $n=18$. Fig. 4- Anafase I de Biscutella lyrata L., $n=8$. Fig. 5- Anafase I de Psychine stylosa Desf. var. typica Maire, $n=15$. Fig. 6- Anafase I de Ilexaquifolium L., $n=20$. Fig. 7- Diacinesis de Peganum harmala L., $n=12$. 


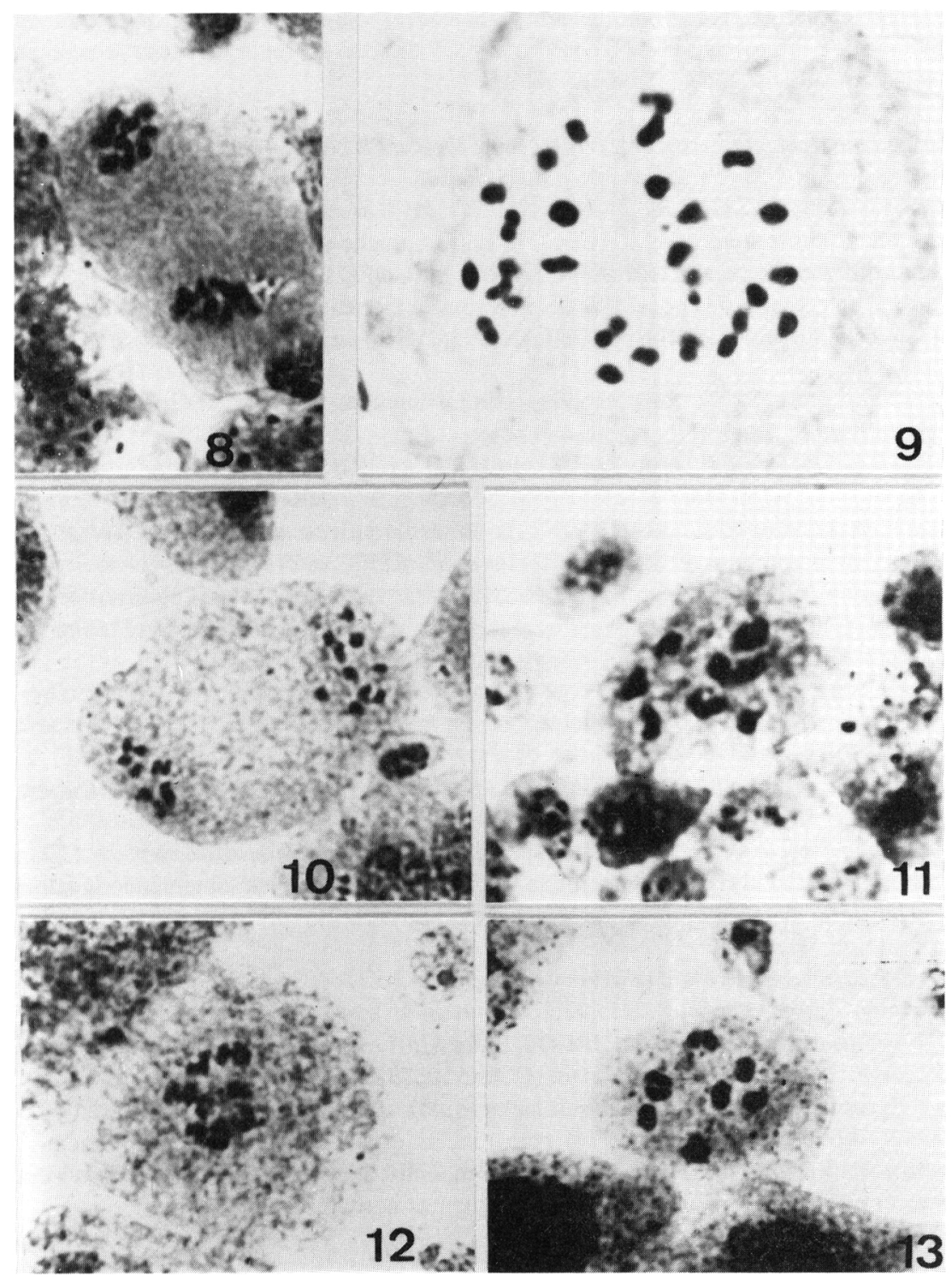

Fig. 8- Anafase I de Stachys circinata L'Hér., $n=10$. Fig. 9- Metafase I de Digitalis purpurea L. subsp. purpurea, $n=28$. Fig. 10- Anafase I de Clinopodium vulgare L. subsp. arundanum (Boiss.) Nyman, $n=10$. Fig. 11-Metafase I de Antirrhinum ramosissimum Cosson \& Durieu, $n=8$. Fig. 12-Diacinesis de Putoria calabrica (L.fil.) DC., $n=11$. Fig. 13-Diacinesis de Calendula stellata Cav., $n=7$. 
Cabezudo, E. Domínguez, E. Ruiz de Clavijo et al. (COFC 19869).

Los numerosos recuentos que conocemos de esta especie se han realizado en material procedente de distintos países del S de Europa y SW de Asia. En todos los casos se ha obtenido este mismo resultado.

Tuberaria macrosepala (Cosson) Willk., Icon. Descr. Pl. Nov. 2: 80 (1859)

Helianthemum guttatum var. macrosepalum Cosson $n=18$ (fig.3)

Material estudiado. MARRUECOS: Entre Larache y El Ksar el Kbir, 8-V-1989, B. Cabezudo, E. Domínguez, E. Ruiz de Clavijo et al.(COFC 19870).

Especie distribuida por el SW de la Península Ibérica y NW de África, de la que existe un único recuento previo, hecho por Gallego (1985) en material ibérico (Cádiz), con el mismo resultado: $\mathrm{n}=18$.

Biscutella lyrata L., Mantissa Alt. 254 (1771)

$B$. didyma subsp. lyrata (L.) Murbeck

$n=8$ (fig.4)

Material estudiado. MARRUECOS: Entre Tiouririne y Ain-Leuh, 9-V-1989, B. Cabezudo, E. Domínguez, E. Ruiz de Clavijo et al. (COFC 19871).

Manton (1932) en material de Sicilia, y Jaretzky (1932, sec.Fedorov, 1969:165), estudiaron la cariología de este taxon distribuido por el occidente de la Región Mediterránea, encontrando en ambos casos el mismo resultado indicado por nosotros.

Psychine stylosa Desf. var. typica Maire, Cat. Pl. Maroc 227 (1932)

$n=15$ (fig.5)

Material estudiado. MARRUECOS: Entre Fes y Birtam-tam, 11-V-1989, B. Cabezudo, E. Domínguez, E. Ruiz de Clavijo et al. (COFC 19872).

El único recuento previo de esta especie endémica del NW de África se debe a GómezCampos (1980). Estudió concretamente la var. maroccana Murbeck, obteniendo igualmente $n=15$ a partir de material también de Marruecos (Marrakech).

Ilex aquifolium L., Sp. P1. 125(1753)

$n=20$ (fig.6)

Material estudiado. MARRUECOS: Entre Ain-Leuh y Ajabo, 9-V-1989, B. Cabezudo, E. Domínguez, E. Ruiz de Clavijo et al. (COFC 19873).

El resultado obtenido coincide con el que indica Maude (1940) para material de Gran Bretaña, y Löve \& Kjellqvist (1974) para plantas españolas (Sierra de Albarracín). Sin embargo, Wulf (1939, sec. Fedorov, 1969:48) encontró $2 n=46$ en plantas de origen alemán. $\mathrm{Al}$ parecer es el primer recuento realizado en plantas norteafricanas.

Peganum harmala L., Sp. Pl. 444 (1753)

$\mathrm{n}=12$ (fig. 7 )

Material estudiado. MARRUECOS: Entre Ait-Messaoud y Ar-Rachidia, desfiladero de N'zala, 9-V-1989, B. Cabezudo, E. Domínguez, E. Ruiz de Clavijo et al. (COFC 19874).

Diversos autores han estudiado la cariología de esta especie distribuida por las regiones Irano-Turánica y Mediterránea. Warburg (1938, sec. Fedorov, 1969:727); Baquar et al. (1965) en material de La India; Lorenzo Andreu \& García Sanz (1950) en material español 
(Aragón), y Singh (1984), en plantas procedentes también de La India correspondientes a la var. stenophyllum Boiss., llegaron a este mismo resultado. Negodi (1937. sec. Fedorov, 1969:727) encontró, no obstante, $2 n=22$.

Stachys circinata L'Hér., Stirp. Nov. 51 (1786)

$n=10$ (fig. 8 )

Material estudiado. MARRUECOS: Tetouan, repetidor de TV de Beni Madem, 500 m.s.m., 6-VI-1989, E. Domínguez, E. Fernández-Galiano, E. Ruiz de Clavijo et al. (COFC 19875).

Especie distribuida por el S de España y NW de África, de la que existe un único recuento anterior, hecho por Ruiz de Clavijo \& Galán (1984) en plantas españolas (Málaga), llegando a este mismo resultado.

Clinopodium vulgare L. subsp. arundanum (Boiss.) Nyman, Consp. 587 (1881) $n=10$ (fig.9)

Material estudiado. MARRUECOS: Entre Mokrisset y Zoumi, 7-VI-1989, E. Domínguez, E. Fernández-Galiano, E. Ruiz de Clavijo et al. (COFC 19876).

Taxon de distribución mediterránea, cuya cariología ha sido estudiada previamente por: Bothmer (1967, sec. Moore, 1982:193) en material de Grecia; Van Loon (1980) en material también de Grecia, y, Papes \& Silic (1981) en plantas yugoslavas. En todos los casos han indicado $2 n=20$.

Antirrhinum ramosissimum Cosson \& Durieu, Bull. Soc. Bot. France II:254 (1855) $n=8$ (fig. 10)

Material estudiado. MARRUECOS: Entre Erfoud y Merzouga, 10-V-1989, B. Cabezudo, E. Domínguez, E. Ruiz de Clavijo et al. (COFC 19877).

Endemismo sahariano estudiado por Baur en 1932, y por Reese en 1957 (sec. Fedorov, 1969:671), quienes asimismo cuentan $2 n=16$.

Digitalis purpurea L., Sp. Pl. 621 (1753) subsp. purpurea $n=28$ (fig. 11)

Material estudiado. MARRUECOS: Entre Cherefat y Bab-Berred, falda del JbelTisirene, 8-VI-1989, E. Domínguez, E. Fernández-Galiano, E. Ruiz de Clavijo et al. (COFC 19878).

Nuestro resultado coincide con el que indican la mayoría de los numerosos autores que han estudiado la cariología de este taxon en material de distintas procedencias.

Putoria calabrica (L.fil.)DC., Prodr. 4:577 (1830)

Asperula calabrica L. fil.

P. hispanica Boiss. \& Reuter

$n=11$ (fig. 12)

Material estudiado. MARRUECOS: Entre Mokrisset y Zoumi, 7-VI-1989, E. Domínguez, E. Fernández-Galiano, E. Ruiz de Clavijo et al. (COFC 19879).

Strid (1971) en plantas de Albania, y Fagerlind (1934, sec. Fedorov, 1969:652) en material cuyo origen desconocemos, estudiaron esta especie de distribución mediterránea obteniendo igualmente, $2 \mathrm{n}=22$. 
Calendula stellata Cav., Icon. Descr.1:3 (1791)

C.algeriensis Boiss. \& Reuter

$n=7$ (fig. 13)

Material estudiado. MARRUECOS: Entre Tiouririne y Ain-Leuh, 9-V-1989, B. Cabezudo, E. Domínguez, E. Ruiz de Clavijo et al. (COFC 19880).

Especie distribuída por Sicilia y norte de África de la que se han realizado varios recuentos todos ellos en material africano. Citaremos a Heyn et al. (1974) y Talavera et al. (1984), quienes utilizaron material marroquí, y Aparicio (1989) en plantas de Argelia. Nuestro resultado coincide con los aportados por estos autores.

\section{BIBLIOGRAFÍA}

APARICIO, A. -1989- Números cromosomáticos de plantas occidentales, 487-507. Anales Jard. Bot. Madrid, 45(2):483-494.

BAQUAR, S.R., S. AKHTAR \& A. HUSAIN -1965- Meiotic chromosome numbers in some vascular plants of Indus Delta.II. Bot. Not., 118,3:289-298.

FEDOROV, A.A. -1969- Chromosome numbers of flowering plants. Leningrad.

GALLEGO, M.J. -1985- Números cromosómicos para la flora española,404-408. Lagascalia, 13(2):311-313.

GÓMEZ-CAMPOS, C. -1980- In A.Löve (ed.) IOPB Chromosome number reports, LXVII. Taxon, 29(2/3):350.

HEYN, C.C., O. DAGAN \& B. NACHMAN, -1974- The annual Calendula species: taxonomy and relationships. Israel Journ. Bot., 23:169-202.

LORENZO-ANDREU, A. y P. GARCÍA-SANZ - 1950- Cromosomas somáticos de plantas espontaneas de la estepa de Aragón.II. Anales Estac. Exp. Aula Dei, 2:12-20.

LÖVE, A. \& E. KJELLQVIST -1974- Cytotaxonomy of Spanish plants. IV. Dicotyledons:Caesalpiniaceae-Asteraceae.Lagascalia, 4(2):153-211.

MANTON, I. -1932-. Introduction to the general cytology of the Cruciferae. Ann. Bot., 46:509556.

MAUDE, P.F. -1940-. Chromosome numbers in some British plants. New Phytol., 39:17-32.

MOORE, D.M. -1982-. Flora Europaea Check-List and Chromosome Index. Cambridge.

PAPES, D. \& C. SILIC -1981- In A.Löve (ed.) IOPB Chromosome number reports, LXX. Taxon, 30(1):70.

RUIZ DE CLAVIJO, E. y C. GALÁN - 1984- Números cromosomáticos de plantas occidentales, 261-269. Anales Jard. Bot. Madrid, 40(2):445-450.

SINGH, P. -1984-In A.Löve (ed.) IOPB Chromosome number reports, LXXXV. Taxon, 33(4): 759 .

SNOW, R. -1963- Alcoholic hidrochloric acid-carmine as a stain for chromosome in squasch preparations. Stain Technol., 38: 9-13.

STRID, A. -1971-. Chromosome numbers in some Albanian Angiosperms. Bot. Not., 124: 490496.

TALAVERA, S., J.A. DEVESA y E.F. GALIANO -1984- Notas cariosistemáticas sobre plantas norteafricanas. I. Compositae. Candollea, 39: 271-280.

VAN LOON, J.CHR. -1980- In A.Löve (ed.) IOPB Chromosome number reports, LXIX. Taxon, 29(5/6): 718-720.

(Aceptado para su publicación en junio de 1991)

Dirección del autor: Departamento de Biología Vegetal y Ecología. Facultad de Ciencias. Universidad de Córdoba. 